\title{
Exotic mosquito threats require strategic surveillance and response planning
}

\section{Cameron E Webb ba,b,c and Stephen L Doggett ${ }^{a}$}

a Medical Entomology, NSW Health Pathology, Westmead Hospital, Sydney, Australia

${ }^{b}$ Marie Bashir Institute for Infectious Diseases and Biosecurity, University of Sydney, NSW, Australia

${ }^{c}$ Corresponding author: cameron.webb@health.nsw.gov.au

\section{Article history}

Publication date: December 2016

Citation: Webb CE, Doggett SL. Exotic mosquito threats require strategic surveillance and response planning. Public Health Res Pract. 2016;26(5):e2651656. doi: http://dx.doi.org/10.17061/phrp2651656

\section{Key points}

- The New South Wales (NSW) Arbovirus Surveillance and Mosquito Monitoring Program is effective at assessing current endemic mosquito-borne disease risk

- Exotic mosquitoes, such as Aedes aegypti and $A$ e. albopictus, pose significant public health and economic threats to NSW

- Exotic mosquito surveillance requires targeted technologies, and incursions may not be detected by existing programs outside of airports and seaports

- Strategic response planning is required to ensure exotic mosquitoes do not become established in NSW

\begin{abstract}
Mosquito-borne diseases caused by endemic pathogens such as Ross River, Barmah Forest and Murray Valley encephalitis viruses are an annual concern in New South Wales (NSW), Australia. More than a dozen mosquito species have been implicated in the transmission of these pathogens, with each mosquito occupying a specialised ecological niche that influences their habitat associations, host feeding preferences and the environmental drivers of their abundance.
\end{abstract}

The NSW Arbovirus Surveillance and Mosquito Monitoring Program provides an early warning system for potential outbreaks of mosquito-borne disease by tracking annual activity of these mosquitoes and their associated pathogens. Although the program will effectively track changes in local mosquito populations that may increase with a changing climate, urbanisation and wetland rehabilitation, it will be less effective with current surveillance methodologies at detecting or monitoring changes in exotic mosquito threats, where different surveillance strategies need to be used.

Exotic container-inhabiting mosquitoes such as Aedes aegypti and Ae. albopictus pose a threat to NSW because they are nuisance-biting pests and vectors of pathogens such as dengue, chikungunya and Zika viruses. International movement of humans and their belongings have spread these mosquitoes to many regions of the world. In recent years, these two mosquitoes have been detected by the Australian Government Department of Agriculture and Water Resources at local airports and seaports. To target the detection of these exotic mosquitoes, new trapping technologies and networks of surveillance locations are required. Additionally, incursions of these mosquitoes into urban areas of the state will require strategic responses to minimise substantial public health and economic burdens to local communities.

\section{Introduction}

Mosquito populations in New South Wales (NSW) pose a risk to human health. Mosquitoes are associated with a wide range of habitats, but relatively few species pose substantial health risks. Some species that bite humans 
have been implicated in the transmission of pathogens including Ross River virus, Barmah Forest virus and Murray Valley encephalitis virus. ${ }^{1}$

The most commonly reported mosquito-borne disease is caused by Ross River virus, and is an illness that is often debilitating, with symptoms persisting for many weeks to months. Outbreaks can be difficult to predict because of the complex relationships between climatic and environmental factors, the virus, mosquito vectors, reservoir hosts, and immunity levels in the human population. ${ }^{2}$ In the absence of broadscale mosquito control in NSW, promotion of personal protection measures by local health authorities, informed by surveillance of mosquitoes and mosquito-borne pathogens, is critical in reducing public health risks. ${ }^{3}$

\section{Current mosquito and mosquito- borne disease surveillance}

The NSW Arbovirus Surveillance and Mosquito Monitoring Program (ASMMP) combines mosquito collection and testing with a sentinel chicken program to detect changes in the abundance of mosquitoes and activity of mosquito-borne viruses. ${ }^{4}$ The abundance of mosquitoes and presence of viruses inform decisions by local health authorities to release public health warnings and take other measures to promote personal protection against mosquitoes. ${ }^{3}$

Mosquitoes are collected on a weekly basis at multiple sites across the state between November and May each year using carbon dioxide-baited encephalitis virus surveillance (EVS) traps. ${ }^{5}$ These traps have been shown to be effective in measuring the abundance of a wide range of local mosquito species of public health importance. ${ }^{6}$ Although current ASMMP methodologies are optimised for the vectors and viruses currently present in NSW, the choice of trap type and surveillance network are not optimal to monitor incursions of exotic mosquitoes because of the different behaviours of these species.

\section{The threat of exotic mosquitoes}

Ae. aegypti and Ae. albopictus are nuisance-biting pests and vectors of dengue virus, chikungunya virus and Zika virus. These mosquitoes are currently not present in NSW. ${ }^{7,8}$ Monitoring for exotic mosquitoes is currently undertaken at first points of entry (i.e. airports and seaports with international arrivals) by the Australian Government Department of Agriculture and Water Resources (DAWR). As these mosquitoes are closely associated with water-holding containers, rather than wetlands, they pose a potentially significant risk to urban areas, where their establishment would significantly increase the threat of arboviral disease to the community.

Ae. aegypti was once present in NSW, including metropolitan Sydney, although the distribution of the species retracted after World War II. The last confirmed detection of Ae. aegypti outside airports or seaports was in 1948 and, although speculation has surrounded the factors contributing to retreat of the mosquito, a combination of social, economic and cultural factors is likely to be responsible. ${ }^{9}$ Local transmission of dengue virus occurred as far south as the NSW central coast. Apart from alpine regions, much of NSW may be able to sustain populations of Ae. aegypti, and the northern regions remain especially vulnerable to re-establishment. ${ }^{9}$ There is also growing concern that insecticide resistance is developing in exotic mosquitoes, with the implication that controlling incursions may become increasingly difficult. ${ }^{10}$

Of increasing concern is the threat that Ae. albopictus poses to NSW. This mosquito has never been established in NSW, but DAWR has detected them at a number of Australian airports and seaports in recent years (including at Sydney International Airport in 2016), indicating that pathways to introduction exist. ${ }^{8}$ This mosquito is more tolerant of cooler climates than Ae. aegypti and distribution modelling has indicated this species could become established in NSW. ${ }^{11}$ In addition to being a vector of mosquito-borne pathogens, Ae. albopictus is a severe nuisance-biting pest, and the economic costs of mosquito control are significant. ${ }^{12}$

Although there has not been a confirmed case of locally acquired dengue virus in NSW for almost 80 years, imported cases of dengue virus ${ }^{13}$, as well as chikungunya virus and Zika virus, infection are reported each year, indicating that there is a pathway for infective individuals to trigger local outbreaks should populations of Ae. aegypti or Ae. albopictus become established.

\section{Strategic surveillance and response planning}

As noted, current mosquito surveillance in NSW is less likely to detect the incursion of Ae. aegypti or $A e$. albopictus, and a strategic surveillance and response plan is required.

Although the EVS traps currently used are effective in monitoring endemic mosquitoes, alternative technologies are required for exotic species. A range of traps targeting egg-laying mosquitoes (i.e. ovitraps) have been used for exotic mosquito monitoring ${ }^{8}$, but there is growing consensus that Biogents' BG-Sentinel (BGS) traps are more sensitive at detecting exotic species. ${ }^{14}$ These commercially produced traps were specifically developed to target these exotic mosquitoes, which are primarily active during the day and are less likely to be detected by the EVS traps used in the ASMMP. ${ }^{15}$ As a consequence, any routine surveillance (e.g. in regions identified as being at high risk of exotic mosquito incursion), port detection response (e.g. enhanced surveillance around airports beyond the range of DAWR surveillance, prompted by the detection of exotic mosquitoes) or incursion detection (e.g. detection of exotic mosquitoes 
outside a DAWR surveillance zone) should be based on the deployment of BGS as opposed to EVS traps.

Surveillance networks will also differ along with trapping technologies. Although the ASMMP primarily targets wetland mosquitoes, with traps located between local human populations and nearby mosquito habitats, traps for exotic mosquito surveillance must be deployed within suburban areas. This shift in focus involves new operational challenges and a need for enhanced community engagement policies.

An exotic mosquito incursion in NSW (i.e. detection outside of the port areas of DAWR responsibility) would require a multistakeholder response to ensure that populations of these mosquitoes do not become established. Establishing formal arrangements with all stakeholders with clearly defined roles and responsibilities for incursion response is critical in preventing the establishment of exotic mosquitoes in Australia. ${ }^{8}$ Such a response should be directed by the NSW Ministry of Health in cooperation with medical entomologists, local government, and other state and federal government agencies, notably the National Arbovirus and Malaria Advisory Committee (under the auspices of the Australian Government Department of Health). The response would include a range of site-specific mosquito surveillance strategies and a specialised mosquito control program. ${ }^{16}$ Given the success of Queensland authorities in controlling populations of exotic mosquitoes, much could be learnt from their experiences. ${ }^{17}$

An integrated approach to the control of containerinhabiting mosquitoes will be required, encompassing larval habitat reduction and targeted insecticide application. Door-to-door surveys of potential 'backyard' habitats will be required. A strong emphasis on community engagement is fundamental to achieve any success. Capacity building within local authorities to rapidly respond to incursions will be critical to reduce the risk of exotic mosquitoes becoming established.

\section{Summary}

An ongoing annual threat to human health posed by endemic mosquitoes and mosquito-borne disease exists in NSW. Although much discussion centres on the influence of climate change in influencing future mosquito-borne disease threats, it is the global movement of humans and their belongings, and the international trade in goods that poses the greatest risk to NSW through the potential introduction of exotic mosquitoes. As evidenced by multiple detections at major ports around the nation, it is clear that pathways exist for the introduction of exotic mosquitoes, as well as exotic and mosquito-borne pathogens. Strategic surveillance and response planning is critical for NSW, and throughout Australia, and should define the roles and responsibilities of local authorities to minimise the potential for exotic mosquito establishment.

\section{Acknowledgements}

The Department of Medical Entomology, NSW Health Pathology, receives funding from the NSW Ministry of Health to coordinate the NSW ASMMP. This manuscript was prepared with the support of the Marie Bashir Institute for Infectious Diseases and Biosecurity, and the National Health and Medical Research Council Centre of Research Excellence in Emerging Infectious Diseases.

\section{Competing interests}

The Department of Medical Entomology, NSW Health Pathology, has been commissioned by a range of companies that develop and market insecticides and insect repellents to test these products and to give expert advice on biting insect biology and disease risks. No external funding was received from these companies for the preparation of this manuscript.

\section{Author contributions}

Both authors contributed to the paper's conception and made substantial intellectual contributions to draft versions.

\section{References}

1. Webb CE, Doggett SL, Russell RC. A guide to the mosquitoes of Australia. Melbourne: CSIRO Publishing; 2016.

2. Claflin $\mathrm{SB}$, Webb CE. Ross River virus: many vectors and unusual hosts make for an unpredictable pathogen. PLoS Pathog. 2015;11(9):e1005070.

3. Webb CE. Are we doing enough to promote the effective use of mosquito repellents? Med J Aust. 2015;202(3):128-9.

4. NSW Health. NSW Arbovirus Surveillance and Mosquito Monitoring Program. Sydney: NSW Health; 2016 [cited 2016 Jun 26]. Available from: medent.usyd.edu.au/ arbovirus/

5. Rohe DL, Fall RP. A miniature battery powered $\mathrm{CO}_{2}$ baited light trap for mosquito-borne encephalitis surveillance. Bull Soc Vector Ecol. 1979;4:24-7.

6. Doggett SL, Russell RC, Clancy J, Haniotis J, Cloonan MJ. Barmah Forest virus epidemic on the south coast of New South Wales, Australia, 1994-1995: viruses, vectors, human cases, and environmental factors. J Med Entomol. 1999;36(6):861-8.

7. Jansen CC, Beebe NW. The dengue vector Aedes aegypti: what comes next. Microbes Infect. 2010;12(4):272-9. 
8. van den Hurk AF, Nicholson J, Beebe NW, Davis J, Muzari OM, Russell RC, et al. Ten years of the Tiger: Aedes albopictus presence in Australia since its discovery in the Torres Strait in 2005. One Health. 2016;2:19-24.

9. Russell RC, Currie BJ, Lindsay MD, Mackenzie JS, Ritchie SA, Whelan PI. Dengue and climate change in Australia: predictions for the future should incorporate knowledge from the past. Med J Aust. 2009;90(5):265-8.

10. Deming R, Manrique-Saide P, Barreiro AM, Cardeña EUK, Che-Mendoza A, Jones B, et al. Spatial variation of insecticide resistance in the dengue vector Aedes aegypti presents unique vector control challenges. Parasit Vectors. 2016;9:67.

11. Hill MP, Axford JK, Hoffmann AA. Predicting the spread of Aedes albopictus in Australia under current and future climates: multiple approaches and datasets to incorporate potential evolutionary divergence. Austral Ecology. 2014;39(4):469-78.

12. Halasa YA, Shepard DS, Fonseca DM, Farajollahi A, Healy S, Gaugler R, et al. Quantifying the impact of mosquitoes on quality of life and enjoyment of yard and porch activities in New Jersey. PloS One. 2014;9(3):e89221.

13. Knope K, Giele C. Increasing notifications of dengue in Australia related to overseas travel, 1991 to 2012. Commun Dis Intel Q Rep. 2013;37(1):E55-9.
14. Baldacchino F, Caputo B, Chandre F, Drago A della Torre A, Montarsi F, Rizzoli A. Control methods against invasive Aedes mosquitoes in Europe: a review. Pest Manag Sci. 2015;71(11):1471-85.

15. Kroeckel U, Rose A, Eiras ÁE, Geier M. New tools for surveillance of adult yellow fever mosquitoes: comparison of trap catches with human landing rates in an urban environment. J Am Mosq Control Assoc. 2006;22(2):229-38.

16. Fonseca DM, Unlu I, Crepeau T, Farajollahi A, Healy SP, Bartlett-Healy K, et al. Area-wide management of Aedes albopictus. Part 2: gauging the efficacy of traditional integrated pest control measures against urban container mosquitoes. Pest Manag Sci. 2013;69(12):1351-61.

17. Queensland Health. Queensland dengue management plan 2015-2020. Brisbane: Queensland Government; 2015 [cited 2016 Sep 1]. Available from: www.health. qld.gov.au/publications/clinical-practice/guidelinesprocedures/diseases-infection/governance/dengue-mgtplan.pdf

\section{Copyright: (c) (i) (2)}

(C) 2016 Webb and Doggett. This article is licensed under the Creative Commons Attribution-NonCommercial-ShareAlike 4.0 International Licence, which allows others to redistribute, adapt and share this work non-commercially provided they attribute the work and any adapted version of it is distributed under the same Creative Commons licence terms. See: www.creativecommons.org/licenses/by-nc-sa/4.0/ 